\title{
Sensor Selection via Closed-Loop Control Objectives
}

\author{
Gary J. Balas, Member, IEEE, and Peter Michael Young, Member, IEEE
}

\begin{abstract}
The ability to stabilize a system and achieve performance objectives using active feedback control is highly dependent on the location, quality, type, and number of control actuators and sensors. One role of a control engineer is to interact with the system designer to locate, size, and determine the quality of actuators and sensors required for effective control. This paper addresses one of these issues: location of sensors based on closedloop objectives. A systematic approach, based on $\mathcal{H}_{2}$ optimal control design techniques, is developed for sensor selection which achieves desired performance objectives and includes system constraints. This approach is applied to the selection of sensors for active vibration attenuation on the NASA Langley Mini-Mast experimental structure.
\end{abstract}

Index Terms - Flexible structures, linear-quadratic control, transducers.

\section{INTRODUCTION}

$\mathbf{T}$ HE ability to stabilize a system and achieve performance objectives using active feedback control is highly dependent on the location, quality, type, and number of control actuators and sensors. One role of a control engineer is to interact with the system designer to locate, size, and determine the quality of actuators and sensors required for effective control. This paper addresses one of these issues: location of sensors based on closed-loop objectives. A systematic approach based on $\mathcal{H}_{2}$ optimal control design techniques is developed for sensor selection which achieves desired performance objectives and includes system constraints, sensor dynamics and noise models. This technique used to select from a candidate list of sensor type and location the optimal set of sensors for vibration attenuation of the NASA Langley Mini-Mast structure.

The placement of sensors and actuators for feedback control has received significant attention the past two decades. One approach to the sensor and actuator placement problem is based on the open-loop system grammians [1]-[5]. These methods make use of the controllability and observability grammians of the system and attempt to optimize the sensor and actuator locations to increase, from a controls perspective, the relative controllability and observability of the important system modes. These grammian based techniques are often applied to flexible structures [1], [2], [3], [5]. The benefit

Manuscript received July 21, 1997; revised June 2, 1998. Recommended by Associate Editor, L. Mestha. This work was supported by NSF under Grant (ECS-9 110254$)$, NASA Langley Control/Structure Interaction Group under Grants (NAG-1-967) and (NAG-1-821), the University of Minnesota McKnight Land-Grant Professorship, and the California Institute of Technology.

G. J. Balas is with the Department of Aerospace Engineering and Mechanics, University of Minnesota, Minneapolis, MN 55455 USA.

P. M. Young is with the Department of Electrical Engineering, Colorado State University, Fort Collins, CO 80523 USA.

Publisher Item Identifier S 1063-6536(99)08544-9. of the open-loop approach to the placement problem is its independence from the type of control design technique to be employed.

Alternatively, the sensor and actuator placement problem has been addressed from minimizing a closed-loop performance metric. This is the approach taken in the paper. Chen and Seinfeld proposed a method for distributed parameter systems where the optimality criterion was given by the space-time integral of the trace of the estimate error covariance [6]. The optimal locations of measurement outputs are computed for a finite set of possible locations using integer programming. Maghami and Joshi propose a method in which the discrete nature of the sensor and actuator placement problem is transformed into a continuous nonlinear programming problem [7]. The authors extend these results in [8] to include two performance criterion: optimize the movement of the transmission zeros of the system into the left half plane and optimize the Hankel matrix corresponding to the controllability and observability grammians in the balanced realization. Skelton et al. extend the results of Chen and Seinfeld for finite dimensional systems [9]-[12]. In Skelton and DeLorenzo [8], [9], an LQG performance metric based on the root mean square contribution of each sensor output is considered [9]. Sensors associated with small cost functions are eliminated. Skelton and Norris extended these results to accommodate noise correlation between sensor and actuator noise sources and sensor and actuator dynamics in [11] and [12]. Juang and Rodriguez use a similar approach to sensor and actuator selection based on LQR and LQG metrics [13]. This paper takes a similar approach to the sensor location problem as Skelton et al. The performance metric to be minimized corresponds to the full information cost in the $\mathcal{H}_{2}$ optimal control problem for a specific set of candidate sensors. The sensor selection problem involves solving an $\mathcal{H}_{2}$ control design problem for each group of sensors to be used for feedback. Closed-loop actuator and sensor placement techniques benefit from the desired control objective being directly accounted for in the placement algorithm. This is in contrast to the open-loop approaches that are independent of the control design.

The NASA Langley Mini-Mast Facility is used as an experimental testbed to verify our approach. The Mini-Mast is an 18-bay 20.16-m long truss beam structure, cantilevered at its base. It is instrumented with six displacement sensors and four accelerometers, which are available for active feedback control. The four accelerometers, and three of the displacement sensor, are located on bay 18. The other three displacement sensor are located on bay 10. In addition, there are six actuators on the structure. The three control actuators are located on 
bay 18 , and the three disturbance actuators are located at bay 9. Section II provides a brief description of the Mini-Mast facility for the context of this work. The vibration attenuation control objectives, performance specifications, and levels of modeling error are also outlined in this section. A more detailed description of the Mini-Mast facility can be found in [14]-[18].

Formulation of the sensor selection problem for the MiniMast structure is described in Section III. The sensor selection problem involves solving an $\mathcal{H}_{2}$ control design problem for each group of sensors to be used for feedback. By exploiting the well-known separation structure of the $\mathcal{H}_{2}$ optimal controller, we are able to quantify the amount of information each group of sensors can observe, in terms of the associated $\mathcal{H}_{2}$ cost of estimating the full system state. This provides an effective means of comparison of different sensor groups. Upon selection of the sensors, an array of controllers are formulated to determine the affect on performance of using a reduced order five-mode model of the Mini-Mast structure as opposed to the higher order 28-mode structural analysis model for control design. Section III-C contains the theoretical analysis and evaluation of these controllers. The sensor selection results indicated that using only bay 18 accelerometers on the Mini-Mast structure for feedback is better for performance than measuring displacements at bay 18 , is as good as measuring bay 10 and 18 displacements, and is almost as good as using every available measurement. These same results are observed on both the full- and reduced order design models.

These results provide insight into the fundamental limitations and achievable levels of performance of the Mini-Mast structure, since they are based on a design technique which guarantees to find the globally optimal controller. Note that they are not intended as practical control designs, since they include no robustness requirement. However, based on these results, $\mu$ synthesis techniques are used to synthesize robust controllers for the Mini-Mast experiment using the four Bay 18 accelerometers for feedback [27], [19], [24], [25]. In addition, the reduced order five-mode model is used in the control problem formulation and the neglected high frequency modes were accounted for via an additive uncertainty model. These controllers were implemented on the Mini-Mast structure. Section IV briefly presents some experimental results from that study, which indicate that high performance control designs were also achievable in practice using only bay 18 accelerometer measurements. Finally in Section V we present some concluding remarks.

\section{The Mini-Mast FACILITY}

The Mini-Mast facility, part of the Control/Structures Interaction Guest Investigator Program, is used to validate the sensor selection approach proposed in this paper. It consists of a deployable and retractable beam truss designed to represent future deployable space trusses [14]-[16]. The Mini-Mast is an 18-bay 20.16-m-long truss beam structure, cantilevered at its base from a rigid foundation. The structural members of the truss are made of a graphite/epoxy composite material with joints composed of titanium and stainless steel. A cable is attached to the tip of the Mini-Mast structure to off load the 350-lb tip mass.

The Mini-Mast facility is instrumented with a total of 51 displacement sensors, three rate gyros (at bay 18), and six accelerometers. For the purposes of this study a subset of these sensors are used, namely the four accelerometers and three displacement sensors located on bay 18, and the three displacement sensors located on bay 10. All ten of these sensors are available for feedback control. In addition, there are six actuators on the structure. Three control actuators are located on bay 18, and three disturbance actuators are located at bay 9. A diagram of the Mini-Mast structure is shown in Fig. 1.

The three torque wheel actuators (TWA's) used for control are located at the tip platform (bay 18) and are mounted in the $x, y$ and $z$ directions. They are denoted TWAX, TWAY and TWAZ, respectively. The TWA transfer functions between voltage and torque are approximately represented by secondorder systems

$$
\frac{36,000 s}{(s+23.5)(s+370)} \text {. }
$$

The torque wheel actuators can saturate since the input voltage is restricted to a range of $\pm 50 \mathrm{~V}$, which effectively limits the current to the motor, and hence the back EMF, limiting the speed to $62 \mathrm{r} / \mathrm{min}$. Disturbance excitations enter the structure via three $50-1 b$ shakers attached at bay 9 of the structure. The shakers are represented as constants in our model since their bandwidth is far above the bandwidth of the system.

Accelerometers located on bay 18, and displacement sensors located on bay 10 and 18, are available for feedback. The accelerometers, Sunstrand QA-1400, have a bandwidth of 0-200 Hz and very low noise characteristics. There are a total of six accelerometers, four of them located on the tip platform (bay 18), and two of them located at the mid-platform (bay 10). There are a total of six Kaman displacement sensors, three each located at bays 10 and 18 . These are mounted on a support structure parallel to the Mini-Mast platform faces and measure displacement normal to the probe. The Kaman sensors have a range of \pm 1 in at bay 10 and \pm 2 in at bay 18 . They are scaled to $\pm 1 \mathrm{mv} / \mathrm{mil}$ and $\pm 0.5 \mathrm{mv} / \mathrm{mil}$ at bays 10 and 18 , respectively.

All the sensor signals are filtered through a third-order 10$\mathrm{Hz}$ Bessel filter (for antialiasing and noise reduction) prior to digitization at $80 \mathrm{~Hz}$. The transfer function for the Bessel filter is

$$
\frac{7.0653 \times 10^{5}}{s^{3}+215.734 s^{2}+1.9514 \times 10^{4} s+7.0653 \times 10^{5}} .
$$

\section{A. Structural Model}

A Nastran finite element model of the Mini-Mast structure was developed and refined by NASA Langley engineers [16]. From this model, a 28 mode, six input, ten output simulation model was synthesized. Both models correlated well with low frequency experimental data. A diagram of the 28-mode six-input ten-output simulation model is shown in Fig. 2. 


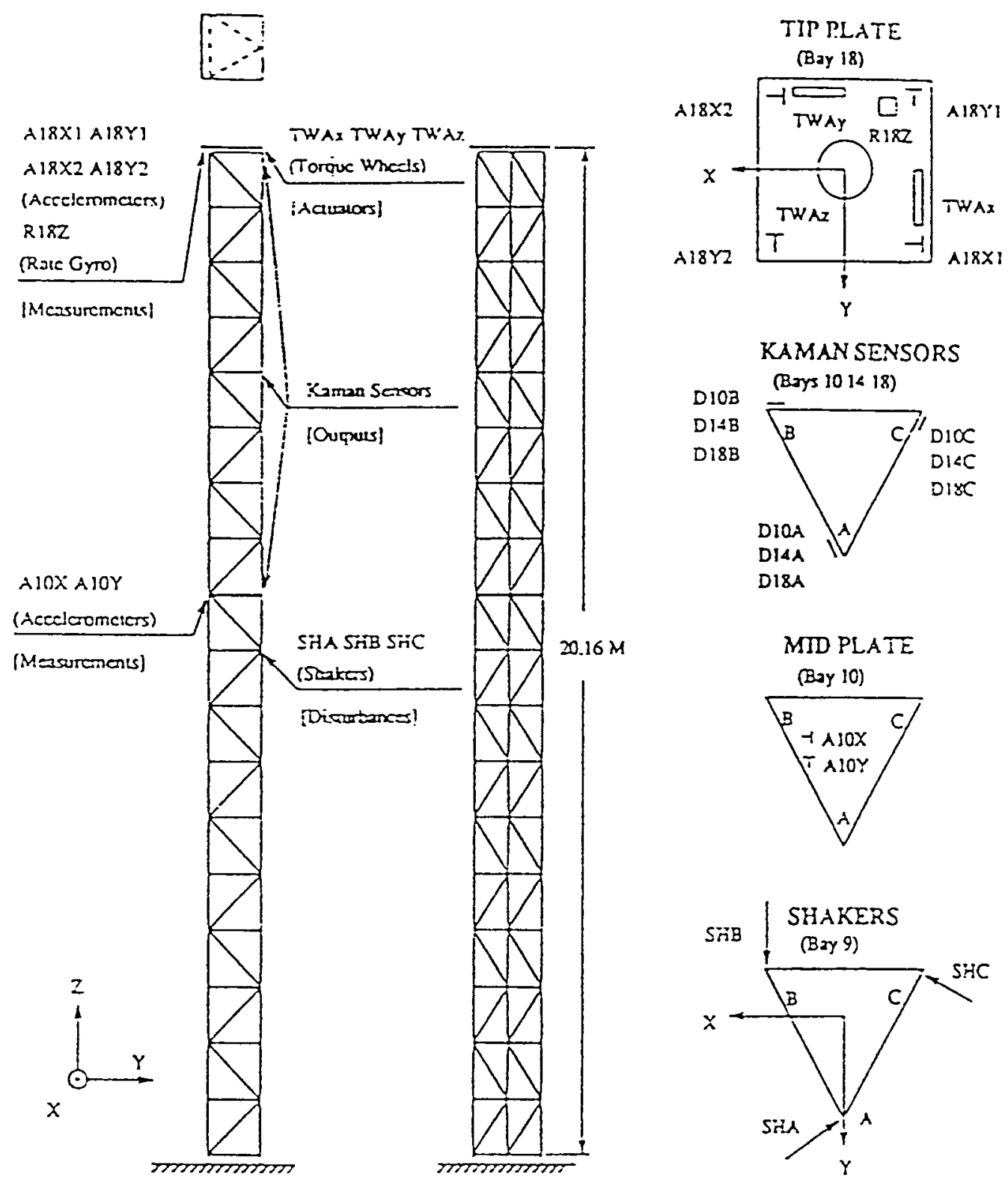

- Tip plaie cabik is na stown.

Fig. 1. Mini-Mast facility with actuator and sensor locations.

The inputs to the structure were the three control actuators and the three disturbance actuators. The output measurements were the six displacement measurements, three each at bays 18 and 10, and the four accelerometers at bay 18. Small deviations were noted between the 28 mode simulation model and physical system. These were attributed to a higher level of damping present in the actual structure compared with the model, because of neglected friction effects.

A reduced order model of the Mini-Mast structure is formulated for control design based on the first five modes of the structure below $45 \mathrm{rad} / \mathrm{s}$. The first five modes, shown in Table I, are to be actively controlled [14], [15], [17], [18]. The remaining 23 modes, between $92.5 \mathrm{rad} / \mathrm{s}$ and 460.8 $\mathrm{rad} / \mathrm{s}$, are accounted for in the control design as unmodeled dynamics. Transfer function magnitude plots of the five- and 28-mode models from the torque wheel actuators and exciters to the bay 18 accelerometers displacement sensors and bay 10 displacement sensors are shown in Figs. 3-5. Note that little error is observed in the natural frequencies of first five modes (which are to be controlled), but of course there is a significant error at high frequency. Hence for practical designs the robustness issue will be very important. This facility provides a fairly realistic complicated problem to test out control ideas in a practical setting.

\section{Feedback Sensor Selection}

The objective of active control on the NASA Langley MiniMast Facility was to attenuate structural vibration at bays 10 and 18 due to external disturbances entering through the exciters at bay 9. Three TWA's were located on the tip of 


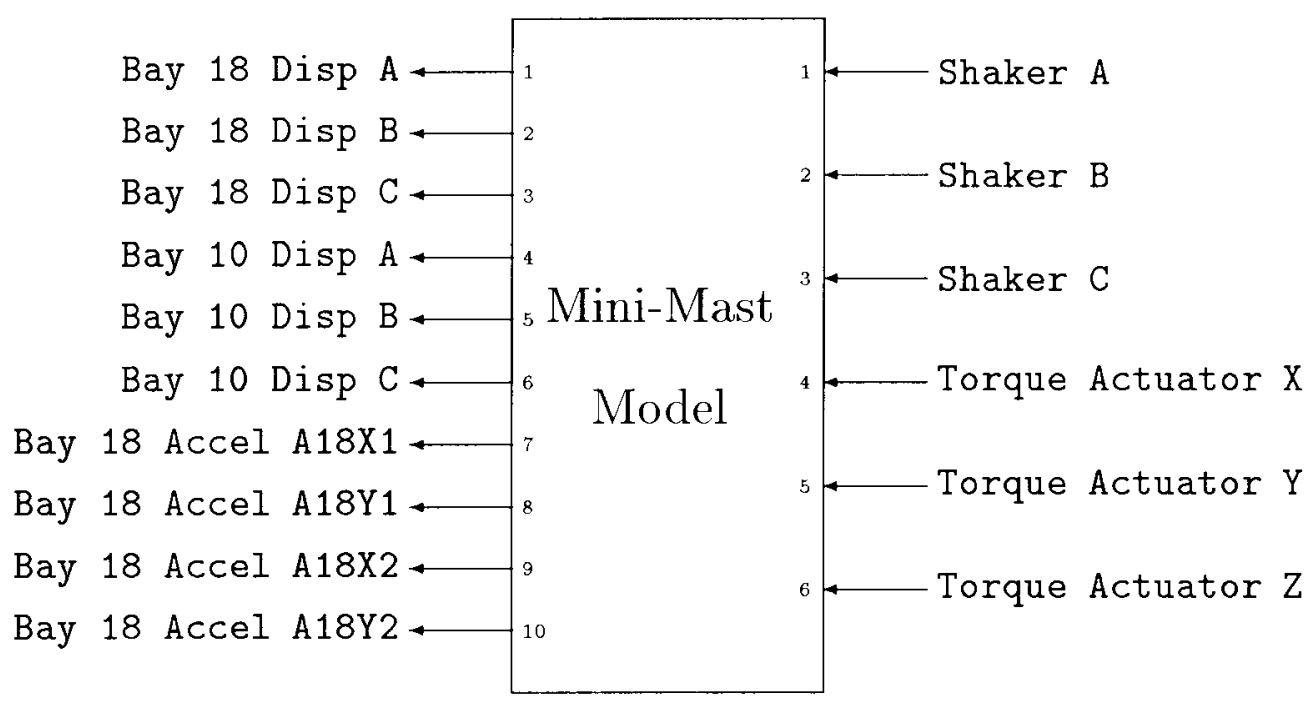

Fig. 2. Mini-Mast control design model.
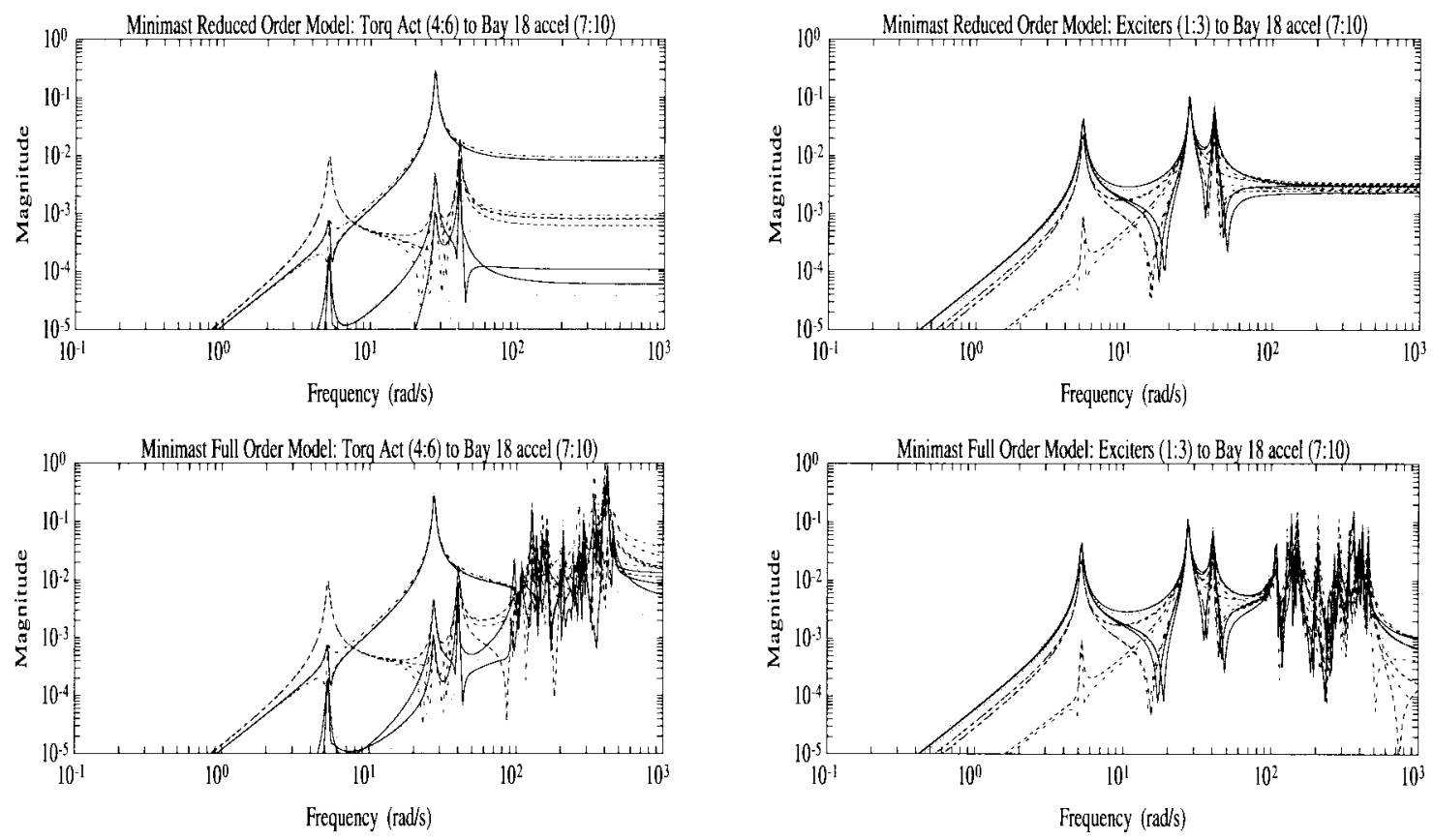

Fig. 3. Transfer functions from torque actuators and exciters to bay 18 accelerometers.

the structure for feedback control. A number of sensors were available for feedback and their selection was left to the discretion of the control engineer. From a systems integration and implementation point of view, it is preferable to use as few sensors for feedback as is necessary to achieve the desired performance objectives. Furthermore, accelerometers are preferred for space applications due to their long history of space flight, ease of placement on the structure, and stand alone capabilities (displacement sensors require a reference point or support structure for measurement).

\section{A. Problem Formulation}

A series of controllers were designed for three different performance objectives and five different feedback sensor configurations in an effort to select the sensor sets to be used for feedback. The three performance objectives were to attenuate the structural response of Mini-Mast at:

- bay 10 and 18 displacement sensors;

- bay 18 displacement sensors only;

- bay 10 displacement sensors only;

subject to external disturbances entering at bay 9 .

The five feedback sensor configurations evaluated were:

- bay 18 accelerometers only;

- bay 18 accelerometers and Bay 18 displacement sensors;

- bay 18 accelerometers, Bay 10 and 18 displacement sensors

- bay 10 and 18 displacement sensors;

- bay 18 displacement sensors. 

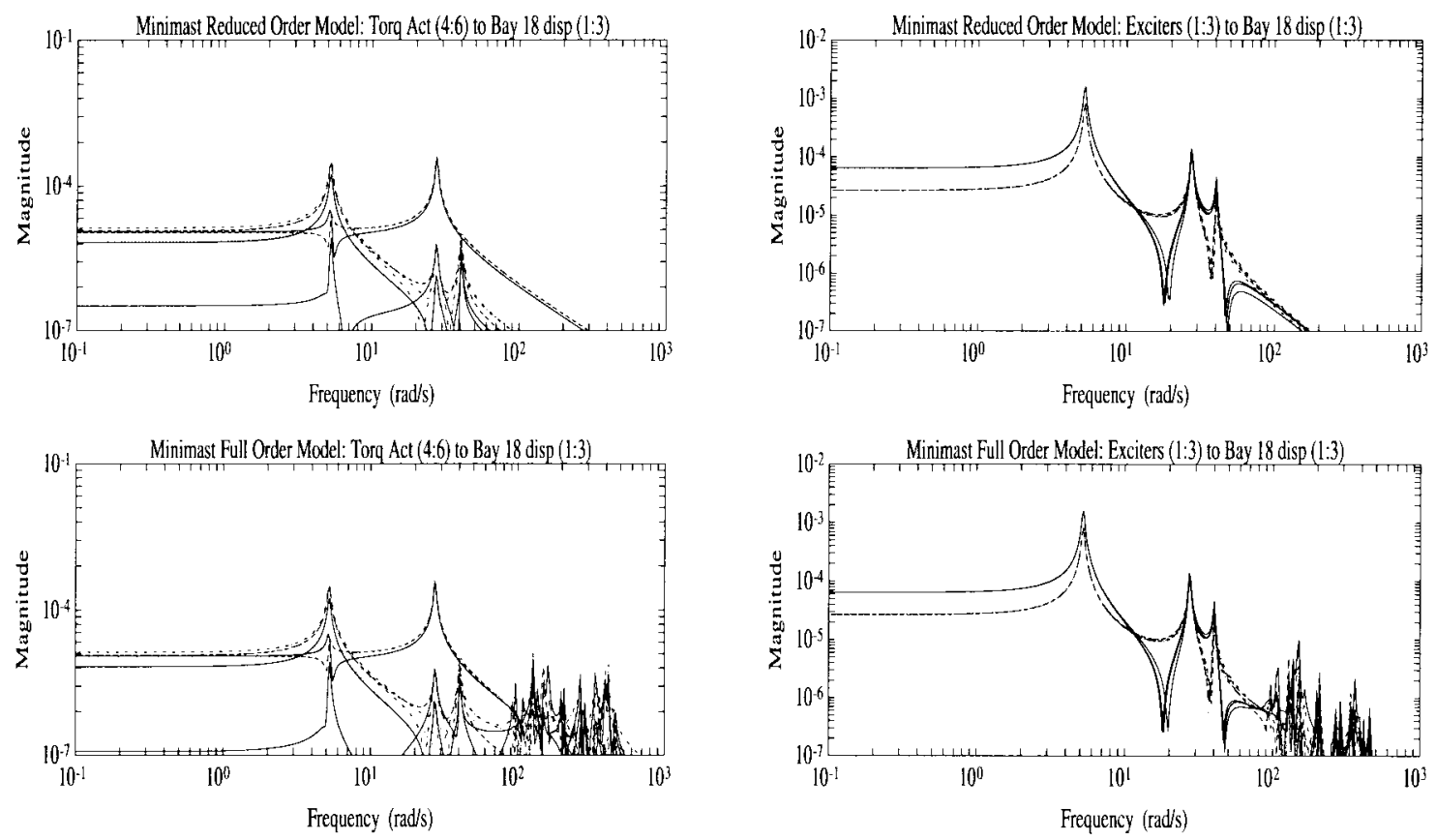

Fig. 4. Transfer functions from torque actuators and exciters to bay 18 displacement sensors.
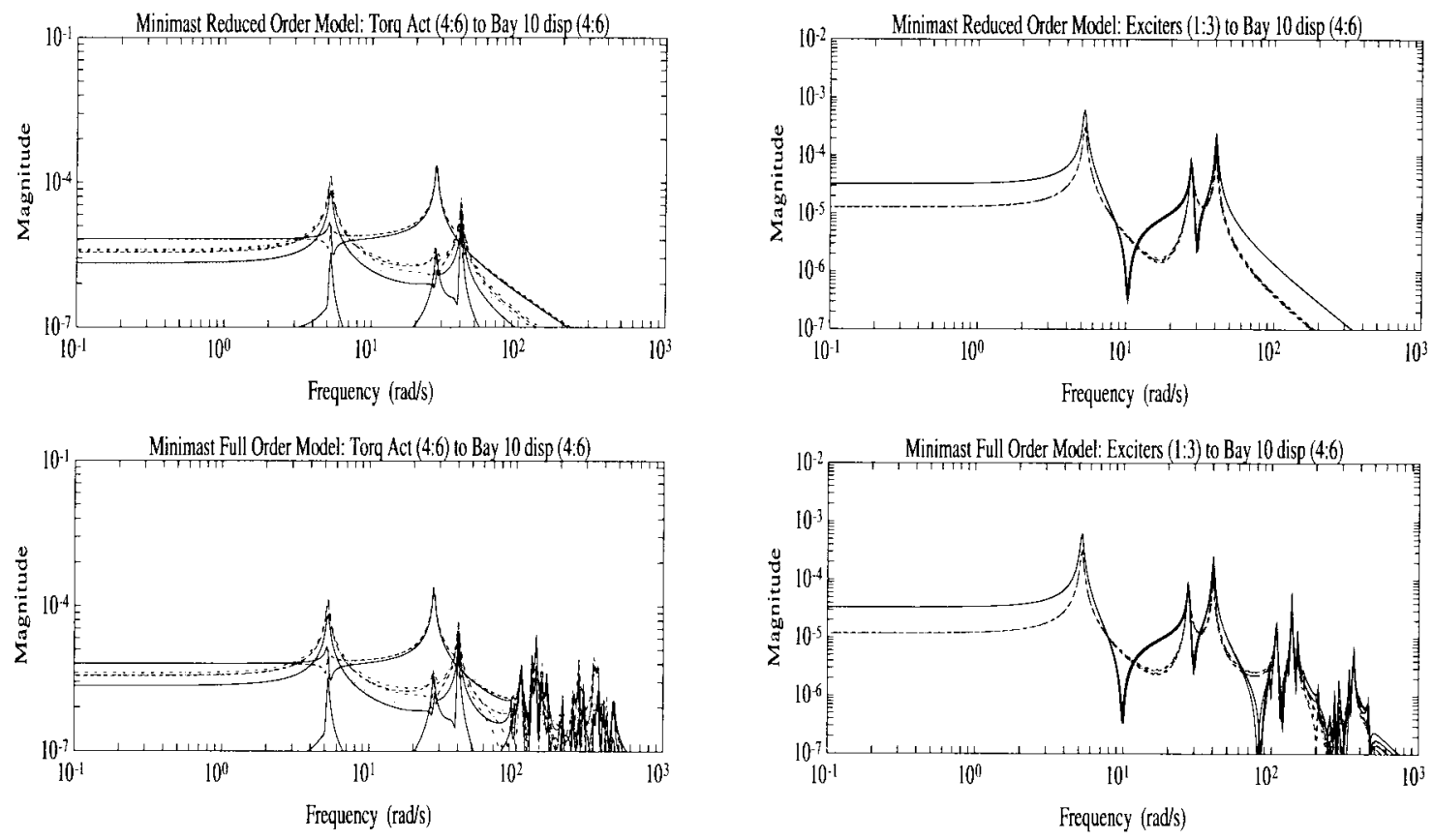

Fig. 5. Transfer functions from torque actuators and exciters to bay 10 displacement sensors.

The following questions were to be determined prior to designing a set of controllers to be tested and implemented on the Mini-Mast Structure.

1) Can accelerometers alone be used as feedback sensors to achieve the performance objective?

2) Was the information content in the measurements sufficient to allow the problem to approach a state feedback control design problem?
3) What is the smallest sensor set we can use without significantly degrading performance?

4) Were the same characteristics for closed-loop control evident on the full-order 28-mode model, and reduced order five-mode control design model?

Questions 1-3 are examined in detail in this section. $\mathcal{H}_{2}$ control design techniques are used to theoretically examine sensor type and placement for the Mini-Mast structure, along with the role of state feedback in the design process. Question 


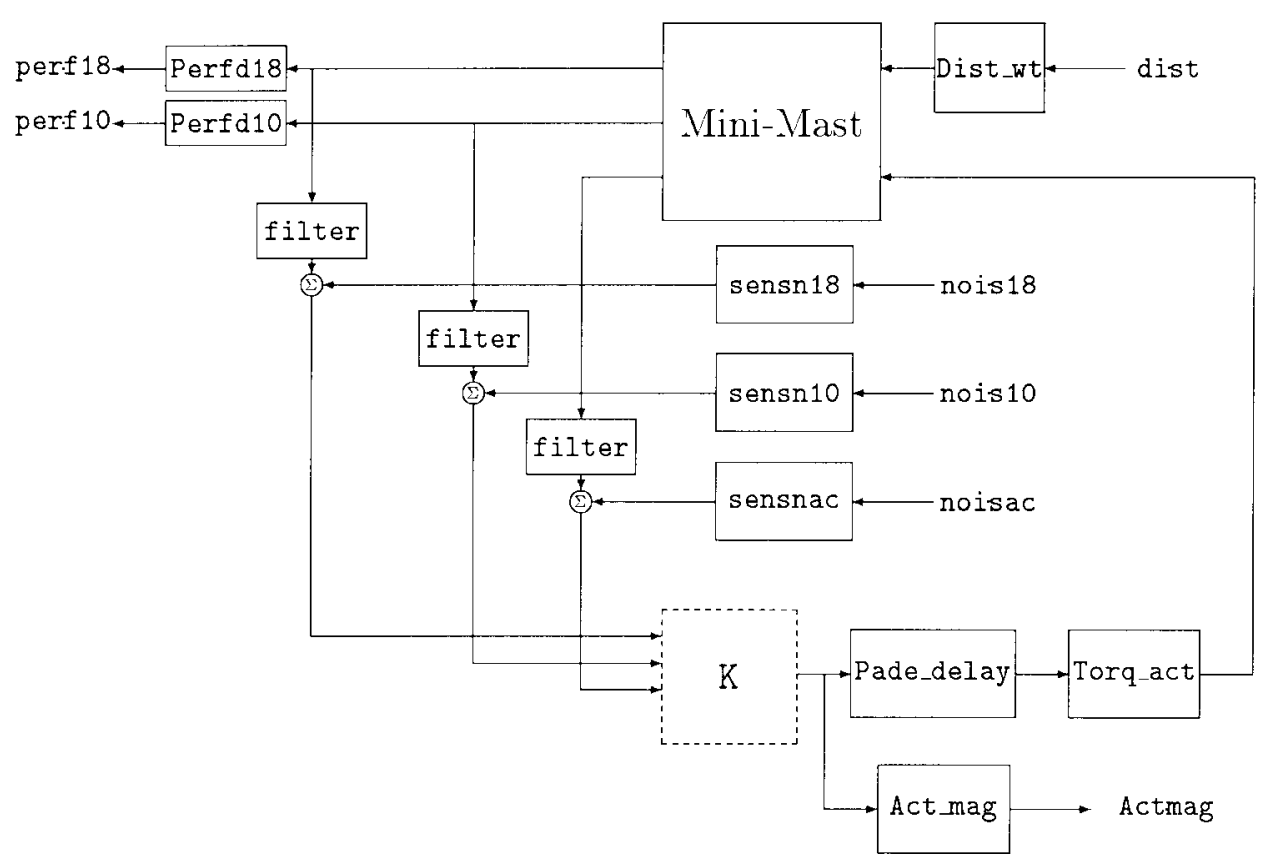

Fig. 6. Interconnection structure for sensor selection.

TABLE I

Natural Frequencies, Damping LeVels and Modes of the Mini-Mast Structure

\begin{tabular}{|c|c|c|c|}
\hline Mode Number & $\begin{array}{c}\text { Predicted } \\
\text { Frequency (rad/sec) }\end{array}$ & $\begin{array}{c}\text { Predicted } \\
\text { Damping }\end{array}$ & $\begin{array}{c}\text { Mode } \\
\text { Description }\end{array}$ \\
\hline \hline 1 & 5.22 & .018 & 1st Y Bending \\
\hline 2 & 5.22 & .018 & 1st X Bending \\
\hline 3 & 27.46 & .012 & 1st Torsional \\
\hline 4 & 40.09 & .010 & 2nd Y Bending \\
\hline 5 & 40.46 & .010 & 2nd X Bending \\
\hline 6 & 92.49 & .005 & Tip Plate \\
\hline 7 & 93.18 & .005 & Diagonal \\
\hline 8 & 96.89 & .005 & 1st Axial \\
\hline 9 & 97.83 & .005 & Tip Plate \\
\hline 10 & 98.02 & .005 & Tip Plate \\
\hline
\end{tabular}

4 is examined with the assumption of no modeling error in the control design process, although of course the issue of robustness arises due to the differences between the 5 and 28 mode models.

These results formed the basis for analysis and synthesis of robust controllers which included modeling error in the problem formulation [17]. These practical designs were implemented on the structure, and extensive experimental results are available to illustrate the high performance achievable with this approach. For reasons of brevity we will focus on the sensor selection problem here, but we refer the interested reader to [17] and [18] for practical controller designs. It is important to note that the theoretical designs presented here were not intended to be implemented on the Mini-Mast facility, because they include no robustness requirements. However they are based on a globally optimal controller, and hence they can be used to examine the fundamental limitations on the achievable performance of a controller. Although they are not intended for practical implementation, they do include models for the actuators, time delays etc., so that a fairly realistic problem is being studied.

The interconnection structure describing the control problem formulation is shown in Fig. 6. The input disturbances to the Mini-Mast structure are 0.1-s 50-N impulses into the exciters. This set of disturbances is represented in the problem formulation by the filter, Dist_wt, whose transfer function is $\frac{15 \tilde{5}}{s+31}$. The sensor noise for the Sunstrand accelerometers and Kaman displacement sensors are very small and taken to be $2 \times 10^{-5}$ for nois18, nois10, and noisac. The controllers will be implemented via a digital computer at a sample rate of $80 \mathrm{~Hz}$. Since the controllers are designed in the analog domain, the sample time delay is accounted for via a first-order Pade delay, whose transfer function representation is $\frac{1-.0125 s}{1+.0125 s}$.

The filter blocks in Fig. 6 represent third-order $10-\mathrm{Hz}$ Bessel filters in line with all the sensor signals. These are anti-aliasing filters which low-pass filter the displacement and accelerometer sensor signals prior to being input to the analog/digital converters, and the controller $K$. The torque actuators are included in the problem formulation via the block Torq_act. Transfer function representations of the filters and torque actuators are provided in Section II. The Mini-Mast structure model shown in Fig. 2, is the six-input ten-output 28-mode model. Comparisons of results between employing the five-mode and 28-mode model will be discussed.

The torque level restrictions on the torque wheel actuators were included into the problem formulation via the Act_mag block. The actuator magnitude weight was selected to correspond with the size of the input disturbances. For impulse disturbances of size $50 \mathrm{~N}$ entering the shakers, the torque wheel actuators will be restricted to a maximum input of $\pm 1 \mathrm{~V}$ 


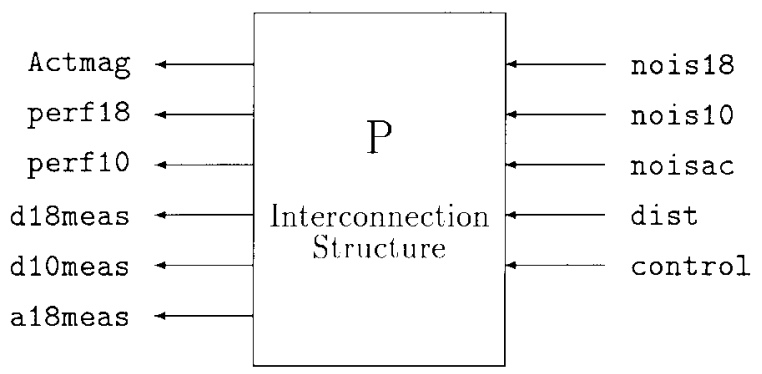

Fig. 7. Control design plant.

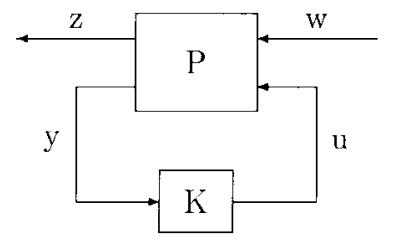

Fig. 8. Linear fractional interconnection.

with the Act_mag scaling set to one. This corresponds to \pm 80 $\mathrm{Nm}$ of torque. The performance objective of attenuating the vibration of the structure was included via weights on the displacement sensors at bay 10 and 18 . The scalings were chosen to have the $\mathcal{H}_{\infty}$ norm of the closed-loop transfer functions between the disturbance inputs dist and the displacement outputs Perfd18, Perfd10 be smaller than one. This leads to selection of Perfd18 to be 1500 and Perfd10 to be 3000 . Measuring performance in terms of damping levels, these performance weights would require the closed-loop system to have approximately 50\% damping of the first three modes. The most general block diagram of the sensor selection problem is shown in Fig. 6 and the interconnection structure used for control design is shown in Fig. 7. Subsets of the diagrams in Figs. 6 and 7 are used in the different control designs.

Note that the $\mathcal{H}_{\infty}$ norm of the disturbance to performance transfer matrix is not used to select feedback sensors. The $\mathcal{H}_{2}$ norm is chosen instead, because of the elegant separation structure available for $\mathcal{H}_{2}$ optimal control. However, $\mathcal{H}_{\infty}$ control design methods were combined with $\mu$-analysis techniques to synthesize robust controllers that would be implemented on the Mini-Mast structure. Therefore it was important to verify that each sensor selection case also achieved similar levels of performance in terms of the $\mathcal{H}_{\infty}$ norm, so as to provide a fair comparison between different sensor groups.

\section{B. $\mathcal{H}_{2}$ Optimal Controller Design}

In this section we will present a very brief review of $\mathcal{H}_{2}$ optimal control, and its connection to LQG/LQR and $\mathcal{H}_{\infty}$ optimal control. Please note that this review is only intended to present the background theory directly relevant to our sensor selection approach. For a more complete and in-depth treatment of these topics we refer the interested reader to [19]-[23] and the references therein.

The canonical setup for $\mathcal{H}_{2}$ (or $\mathcal{H}_{\infty}$ ) optimal control is illustrated in Fig. 8. Note that in general $w, z, y, u$ are all vector signals, so that both the plant $P$ and the controller
$K$ are multiinput-multioutput systems. In this canonical form the exogenous disturbance signals are collected in $w$, and the variables to be controlled are in $z$. The controller has access to measurements $y$ and generates control signals $u$.

The $\mathcal{H}_{2}$ optimal control problem is to find a stablizing real rational controller $K(s)$ which minimizes the $\mathcal{H}_{2}$ norm of the closed-loop system from disturbances $w$ to controlled variables $z$. Thus $K(s)$ should solve

$$
\begin{aligned}
\inf _{K \in \mathcal{K}_{\mathcal{S}}}\left\|T_{z w}\right\|_{2} \\
=\inf _{K \in \mathcal{K}_{\mathcal{S}}}\left(\frac{1}{2 \pi} \int_{-\infty}^{\infty} \operatorname{Tr}\left(T_{z w}^{*}(j w) T_{z w}(j w)\right) d w\right)^{\frac{1}{2}}
\end{aligned}
$$

where $T_{z w}(s)$ is the transfer matrix from $w$ to $z$ in Fig. 8, and $\mathcal{K}_{\mathcal{S}}$ is the set of all real-rational linear time-invariant (LTI) controllers that renders the feedback interconnection internally stable.

The state-space realization of the transfer matrix $P(s)$ is partitioned in accord with Fig. 8 as

$$
P(s)=\left[\begin{array}{c|c}
A & B \\
-- & -- \\
C & D
\end{array}\right]=\left[\begin{array}{c|cc}
A & B_{1} & B_{2} \\
-- & -- & -- \\
C_{1} & D_{11} & D_{12} \\
C_{2} & D_{21} & D_{22}
\end{array}\right] .
$$

In order for the problem to be well posed in general, the following technical assumptions are made (see [22] for details):

1) $\left(A, B_{2}\right)$ is stabilizable and $\left(C_{2}, A\right)$ is detectable

2) $D_{12}$ is full column rank with $\left[D_{12} D_{\perp}\right]$ unitary and $D_{21}$ is full row rank with $\left[\begin{array}{c}D_{21} \\ \tilde{D} \perp\end{array}\right]$ unitary.

3) $D_{11}$ and $D_{22}$ are both zero.

4) $\left[\begin{array}{cc}A-j \omega I & B_{2} \\ C_{1} & D_{12}\end{array}\right]$ has full column rank for all $\omega$.

5) $\left[\begin{array}{cc}A-j \omega I & B_{12} \\ C_{2} & D_{21}\end{array}\right]$ has full row rank for all $\omega$.

Note that both the unitary assumptions in 2), and the $D_{22}$ assumption in 3), are only included to simplify the presentation of results, and can be relaxed. Given these assumptions the problem always has a unique optimal solution, and the controller that minimizes the $\mathcal{H}_{2}$ cost function is given as

$$
K_{\mathcal{H}_{2}}=\left[\begin{array}{c|c}
A+B_{2} F_{2}+L_{2} C_{2} & -L_{2} \\
------ & -- \\
F_{2} & 0
\end{array}\right]
$$

where $F_{2}=-B_{2}^{\prime} X_{2}, L_{2}=-Y_{2} C_{2}^{\prime}$ with $X_{2}$ and $Y_{2}$ being the solutions to the algebraic Riccati equations

$$
\begin{aligned}
X_{2} & : A^{\prime} X_{2}+X_{2} A+X_{2} B_{2} F_{2}+C_{1}^{\prime} C_{1}=0 \\
Y_{2} & : A Y_{2}+Y_{2} A^{\prime}+L_{2} C_{2} Y_{2}+B_{1} B_{1}^{\prime}=0 .
\end{aligned}
$$

These Riccati equations are guaranteed a priori to have a solution. Note also that the $\mathcal{H}_{2}$ optimal control problem is easily shown to be equivalent to linear quadratic Gaussian (LQG) optimal control (see [20] and [21]). This follows by using Parseval's theorem to restate the frequency domain formulation of the $\mathcal{H}_{2}$ norm in the time domain

$$
\begin{aligned}
\left\|T_{z w}\right\|_{2} & =\left(\frac{1}{2 \pi} \int_{-\infty}^{\infty} \operatorname{Tr}\left(T_{z w}^{*}(j w) T_{z w}(j w)\right) d w\right)^{\frac{1}{2}} \\
& =\left(\int_{-\infty}^{\infty} \operatorname{Tr}\left(T_{z w}^{*}(t) T_{z w}(t)\right) d t\right)^{\frac{1}{2} .}
\end{aligned}
$$


Consider now two special cases to Fig. 8, which are labeled as follows [22].

FI: Full information

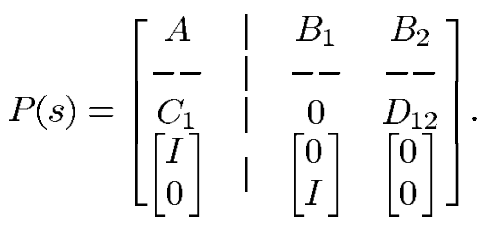

OE: Output estimation

$$
P(s)=\left[\begin{array}{c|cc}
A & B_{1} & B_{2} \\
-- & -- & -- \\
C_{1} & 0 & I \\
C_{2} & D_{21} & 0
\end{array}\right] .
$$

The FI problem derives its name from the fact that the controller is provided with full information since the measurement signal is given as $y=\left(\begin{array}{l}x \\ w\end{array}\right)$, i.e., it contains complete copies of both the disturbance input $w$ and the system state $x$. Thus, in the FI problem, the controller has access to all possible information about the system being controlled. Hence all the control cost is associated with actuating the system, and not observing it, and in fact the globally optimal controller turns out to be simply a constant gain. This situation is reminiscent of the standard state-feedback (SF) problem where $y=x$ (i.e., all states can be measured), also referred to in the $\mathcal{H}_{2}$ case as the linear quadratic regulator (LQR) problem. In fact it can be shown that in the $\mathcal{H}_{2}$ case the measurement of disturbance $w$ is not used, so that the FI and SF problems are indeed equivalent (the relationship for the $\mathcal{H}_{\infty}$ case is more involved).

The motivation for the term output estimation in the $\mathrm{OE}$ problem arises from the fact that the controlled variables are given as $z=C_{1} x+u$. Note that the controller is attempting to minimize the $\mathcal{H}_{2}$ norm of the closed-loop system, and this norm would be set to zero if one could choose $u=-C_{1} x$. Thus, in some sense, the controller generates a control signal $u$ which attempts to estimate the output $-C_{1} x$. The cost of control in this case reflects the cost of estimating that output (note that if the measured state were available, then this cost would be zero). This problem is closely related to the optimal observer or Kalman filtering problem (in the $\mathcal{H}_{2}$ case).

The $\mathcal{H}_{2}$ optimal controller, $K_{\mathcal{H}_{2}}$, has the well-known separation structure, in that the problem is solved by using an optimal observer to estimate the state, and feeding that estimate into an optimal state feedback gain. To be specific $K_{\mathcal{H}_{2}}$ is exactly the optimal output estimator of $F_{2} x$, where $F_{2}$ is the optimal state feedback from the full information problem.

Thus the general problem is solved by combining the FI and OE problems. Moreover, the cost associated with each part of the controller's function (state feedback and state estimation) can be precisely quantified: the squared $\mathcal{H}_{2}$ norm of the closedloop transfer function $T_{z w}$ is equal to the squared $\mathcal{H}_{2}$ norm of the associated full information problem (FI), plus the squared $\mathcal{H}_{2}$ norm of the associated OE. We denote this as

$$
\left\|T_{z w}\right\|_{2}^{2}=\mathrm{FI}^{2}+\mathrm{OE}^{2} .
$$

The $\mathcal{H}_{2}$ controllers in this paper are synthesized using the MATLAB $\mu$ Analysis and Synthesis ( $\mu$-Tools) toolbox [19], which provides an implementation of the above formulas. We again note that these $\mathcal{H}_{2}$ control designs are used to examine fundamental tradeoffs and limitations inherent in the Mini-Mast experiment, and to decide on appropriate choices of sensors and performance requirements. By using $\mathcal{H}_{2}$ (or $\mathcal{H}_{\infty}$ ) designs one is guaranteed to find the globally optimal controller for the particular performance objective. This is very useful in examining system properties and tradeoffs as is done here. We again stress that it is important to note that $\mathcal{H}_{2}$ (or $\mathcal{H}_{\infty}$ ) designs include no robustness considerations, and this must be borne in mind when interpreting the results. In contrast, robust control design methods do account for both uncertainty and performance, but are usually not guaranteed to find the globally optimal controller (and hence their utility for studying fundamental tradeoffs is limited).

\section{Sensor Selection}

For each performance objective and set of feedback sensors the corresponding outputs of the interconnection structure from Fig. 6 were selected and an $\mathcal{H}_{2}$ optimal control design synthesized. For example, the interconnection diagram to design controllers using Bay 18 accelerometers with performance specifications on Bay 10 and 18 is shown in Fig. 9. The separation structure property of the $\mathcal{H}_{2}$ optimal controller was used to calculate the individual components of the optimal $\mathcal{H}_{2}$ cost. In addition the $\mathcal{H}_{\infty}$ norms of the closedloop transfer functions from disturbance-to-performance and from disturbance-to-control were also calculated for reasons outlined earlier.

The results of this analysis are collected in Table II for the full-order model and Table III for the reduced-order model. The nomenclature used is as follows:
TC Total cost of output feedback $\mathcal{H}_{2}$ norm.
FI Full information $\mathcal{H}_{2}$ norm.
OE Output estimation $\mathcal{H}_{2}$ norm.
CM $\mathcal{H}_{\infty}$ norm of disturbance to control magnitude.
DP $\quad \mathcal{H}_{\infty}$ norm of disturbance to displacements.

The selection criteria for our sensor sets and performance criteria were based on these norms. Recall that the total $\mathcal{H}_{2}$ output feedback cost (TC) for the control problem is the square root of the sum of the squared costs of the full information control problem (FI) and the the output estimation problem $(\mathrm{OE})$, denoted here as

$$
\mathrm{TC}^{2}=\mathrm{FI}^{2}+\mathrm{OE}^{2} \text {. }
$$

The FI represents the $\mathcal{H}_{2}$ norm of the system if full state feedback were available. The $\mathrm{OE}$ represents the cost of estimating the modes (states) of the structure to be controlled with an observer. Thus, when the FI cost is large in relation to the $\mathrm{OE}$ cost, the problem is being dominated by the cost of actuating the structure, so that the sensors are providing adequate information. Recall further that the FI cost represents the best $\mathcal{H}_{2}$ control possible, with everything known about the system available for feedback control. Hence, when the FI cost represents a large fraction of the total TC cost, then additional measurements will not help, because the current measurements are already providing close to the pure FI cost. 


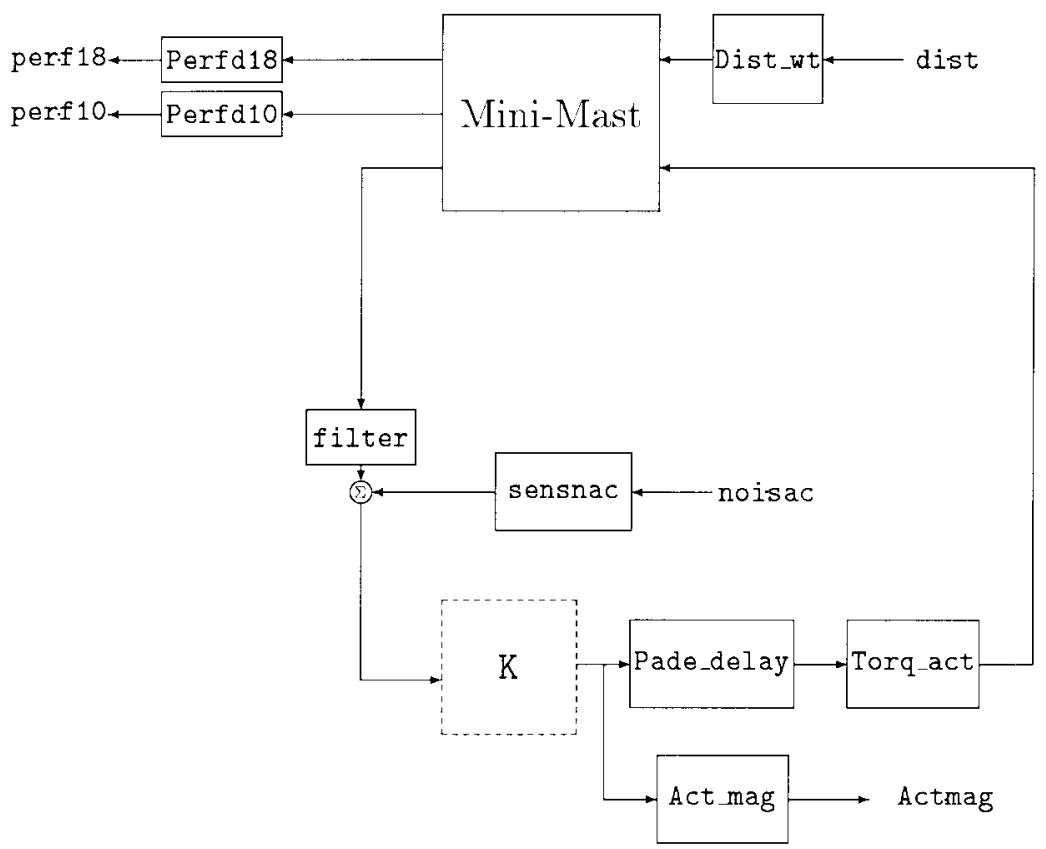

Fig. 9. Interconnection structure for sensor selection using bay 18 accelerometers.

TABLE II

Full-Order Model SEnsor Selection

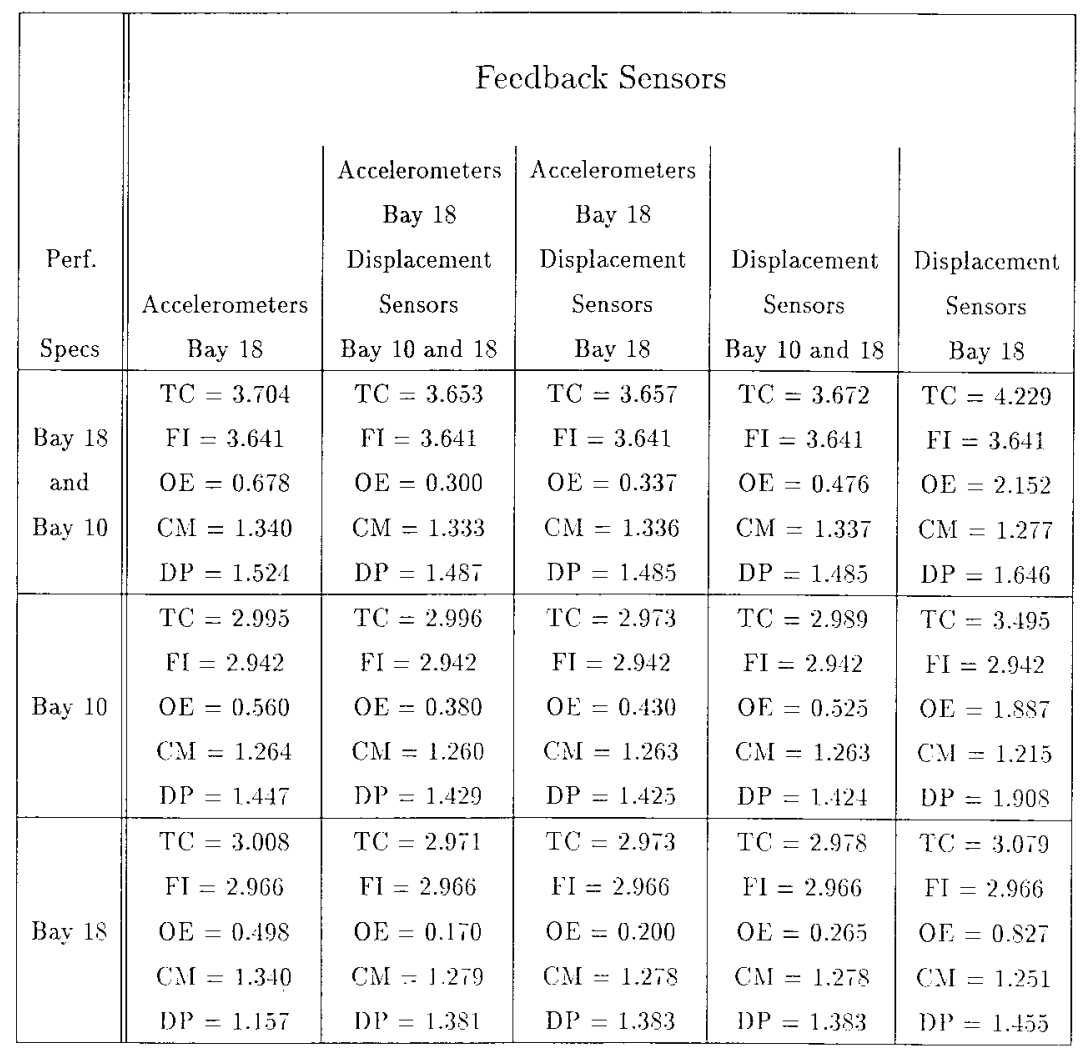

Consider first of all the controllers designed for bay 10 and 18 performance specifications, corresponding to the first row of Table II. Note that the FI cost is constant across any row, because it depends on the performance specification, and not the sensors used (since it assumes the full state is available). The FI cost of 3.641 dominates the $\mathcal{H}_{2}$ cost in the first four control designs of row 1 in Table II, all of which have very similar $\mathcal{H}_{2}$ norms. However, the final sensor set yields a significant $\mathrm{OE}$ cost, and we will return to this point later.

This means that any of the first four sensor sets is providing good measurements for feedback control to achieve high closed-loop performance at both bays 10 and 18. Of course, 
TABLE III

Reduced Order Model Sensor Selection

\begin{tabular}{|c|c|c|c|c|c|}
\hline & \multicolumn{5}{|c|}{ Feedback Sensors } \\
\hline $\begin{array}{l}\text { Perf. } \\
\text { Specs }\end{array}$ & $\begin{array}{c}\text { Accelerometers } \\
\text { Bay } 18\end{array}$ & $\begin{array}{l}\text { Accelerometers } \\
\text { Bay } 18 \\
\text { Displacement } \\
\text { Sensors } \\
\text { Bay } 10 \text { and } 18\end{array}$ & $\begin{array}{l}\text { Accelerometers } \\
\text { Bay } 18 \\
\text { Displacement } \\
\text { Sensors } \\
\text { Bay } 18\end{array}$ & $\begin{array}{l}\text { Displacement } \\
\text { Sensors } \\
\text { Bay } 10 \text { and } 18\end{array}$ & $\begin{array}{c}\text { Displacement } \\
\text { Sensors } \\
\text { Bay } 18\end{array}$ \\
\hline $\begin{array}{l}\text { Bay } 18 \\
\text { and } \\
\text { Bay } 10\end{array}$ & $\begin{aligned} \mathrm{TC} & =3.680 \\
\mathrm{FI} & =3.621 \\
\mathrm{OE} & =0.654 \\
\mathrm{CM} & =1.336 \\
\mathrm{DP} & =1.526\end{aligned}$ & $\begin{array}{l}\mathrm{TC}=3.631 \\
\mathrm{FI}=3.621 \\
\mathrm{OE}=0.275 \\
\mathrm{CM}=1.330 \\
\mathrm{DP}=1.487\end{array}$ & $\begin{aligned} \mathrm{TC} & =3.633 \\
\mathrm{FI} & =3.621 \\
\mathrm{OF} & =0.298 \\
\mathrm{CM} & =1.332 \\
\mathrm{DP} & =1.486\end{aligned}$ & $\begin{aligned} \mathrm{TC} & =3.664 \\
\mathrm{FI} & =3.621 \\
\mathrm{OE} & =0.560 \\
\mathrm{CM} & =1.335 \\
\mathrm{DP} & =1.48 .4\end{aligned}$ & $\begin{array}{l}\mathrm{TC}=4.179 \\
\mathrm{FI}=3.621 \\
\mathrm{OE}=2.08 \overline{7} \\
\mathrm{CN}=1.2 \bar{i} \\
\mathrm{DP}=1.641\end{array}$ \\
\hline
\end{tabular}

column 2, where all the available measurements are used (accelerometers on bay 18, and displacement sensors on bays 10 and 18) yields the lowest $\mathcal{H}_{2}$ norm, but the improvement is insignificant over column 1 , where only accelerometers on bay 18 are used. To be specific, note that column 2 yields a total $\mathcal{H}_{2}$ cost within $0.3 \%$ of the optimal FI cost, whereas column 1 yields a cost within $1.7 \%$ of the optimal FI cost. Hence the $\mathcal{H}_{2}$ cost added by not using the displacement sensors is insignificant in comparison with the cost of controlling the structural modes of the system. From an implementation pointof-view, the expense associated with adding displacement sensors to the bay 18 accelerometers, does not warrant their use as feedback sensors.

Note also the power of the $\mathcal{H}_{2}$ optimal approach taken here. Assuming the problem as stated (i.e., our model of the plant is accurate, and we are interested in the $\mathcal{H}_{2}$ performance objectives stated), then using only accelerometers on bay 18 is less than $2 \%$ off the optimal FI cost. This means that using these sensors one achieves within $2 \%$ of what one could possibly achieve by adding any number/type of sensors at any location. Thus it is clear that these sensors are adequate for the task, and there is very little to be gained by adding additional sensors.

Furthermore, we note that our discussion of $\mathcal{H}_{2}$ optimal control was limited to LTI controllers, because we wanted to state our results in the frequency domain. In fact one can generalize the notion of $\mathcal{H}_{2}$ optimal control, by considering time-domain versions of the appropriate definitions, to consider nonlinear and time-varying control. It turns out that the LTI controller formulas presented earlier are optimal even if one considers nonlinear time-varying control laws (because the plant is LTI). Hence the statements made above are valid even allowing nonlinear time-varying controllers.

Note also that these same trends are noted when the performance specifications are associated with bay 10 and 18 independently (rows 2 and 3 of Table II). It is still true that the first four sensor sets (first four columns) all provide close to the FI cost. Hence all these sensor sets are providing excellent information for feedback control. Furthermore it is again clear that only using the accelerometer measurements at bay 18 (column 1) is providing close to the performance achievable with any combination of sensors.

Note that these results have effectively answered three of the questions posed in Section III-A: accelerometers alone are sufficient as feedback sensors to achieve the desired closed-loop performance; the information content in the measurements approaches a state feedback problem; a sensor set consisting of bay 18 accelerometers only is adequate. However Table II contains a wealth of information beyond these issues. For instance one could consider how effective are accelerometers versus displacement sensors for this problem. Examining the table one finds that using accelerometers on bay 18 outperforms using displacement sensors on bay 18 , and is only very slightly worse than using displacement sensors on bays 10 and 18 (or indeed using all the available sensors). This seems to indicate the superiority of accelerometers over displacement sensors for this problem.

Note also that, as mentioned earlier, using displacement sensors on bay 18 (column 5) is the only place where we see a significant $\mathrm{OE}$ cost. On examining this column it is seen that displacement sensors on bay 18 are able to deliver close to the FI cost when the performance objectives are on bay 18 only. However they are not able to deliver close to the FI cost when the performance objectives are on bay 10, or both bays 10 and 18. This is in contrast to using accelerometers on bay 18 (column 1) which is able to deliver close to the FI cost for all three different performance objectives. Hence for this problem the accelerometers are outperforming the displacement sensors very slightly in the case where sensors and performance objectives are colocated, but the performance difference is much larger (in favor of the accelerometers) in the noncolocated case.

Finally we consider question 4 from Section III-A, and the results for the reduced order five-mode Mini-Mast model are given in Table III. Note that here the higher order dynamics do not enter into the problem. In this case we only consider the most interesting performance objective, that of attenuating vibration at both bays 10 and 18 . It is readily seen that the results for the reduced order five-mode model are essentially the same as those already discussed for the full-order 28- 


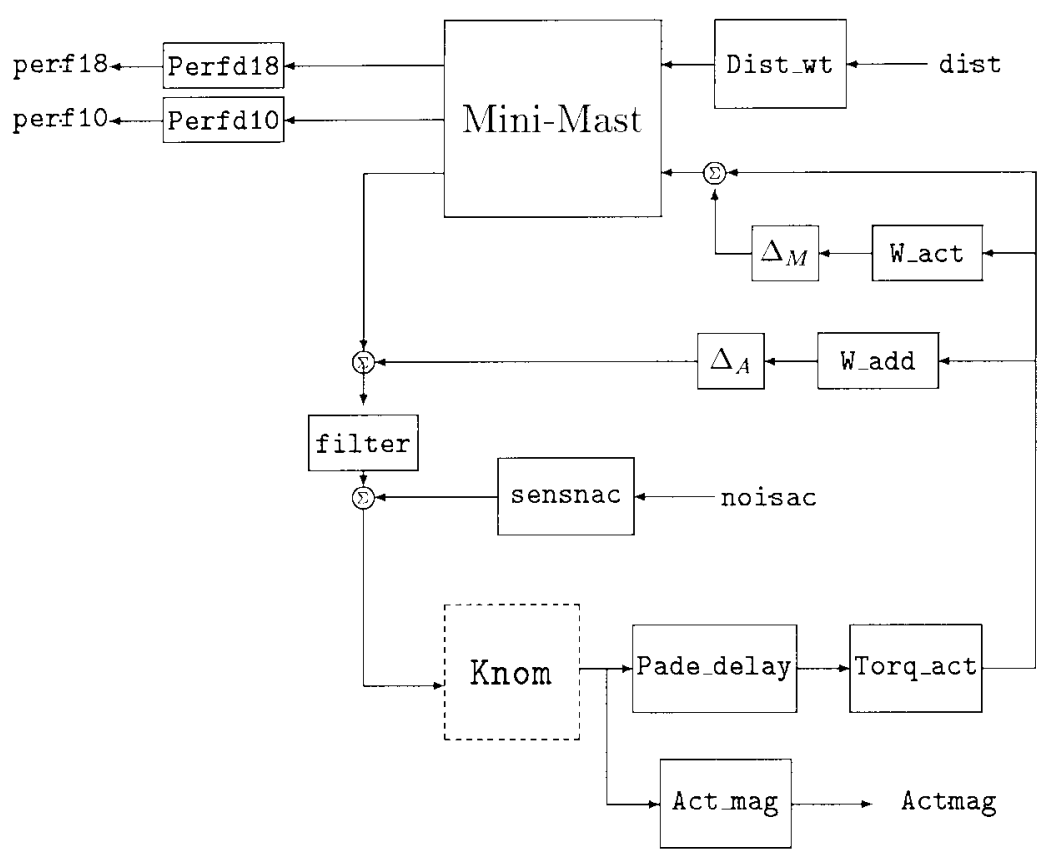

Fig. 10. Interconnection structure for robust control design using bay 18 accelerometers.

mode model. Once again we see that use of accelerometers on bay 18 only for feedback is able to deliver high closed-loop performance on bays 10 and 18. Bay 18 accelerometers lead to almost a full information (state feedback) control design problem with the full information part of the control design problem dominating the output estimation problem. These results imply that, for either the full or reduced order models, one is able to easily observe all the information required for control design and the difficult part is controlling the system via the torque wheel actuators.

The disturbance-to-control (CM) and disturbance-todisplacement (DP) transfer function $\mathcal{H}_{\infty}$ norms of the five controllers, shown in Tables II and III, are all similar. These results indicate that the five controllers achieve a consistent level of actuator torque and performance levels in an $\mathcal{H}_{\infty}$ norm sense as well. The similarity of $\mathcal{H}_{\infty}$ norms provide additional confirmation that the $\mathcal{H}_{2}$ control design results will be valid when controllers are designed to minimize the $\mathcal{H}_{\infty}$ norm between the inputs and outputs.

The methodology proposed here for sensor selection, based on $\mathcal{H}_{2}$ optimal control, will be most suitable when the achievable robust performance level is close to the optimal nominal performance of the system. In other words, these results are extendible to the actual Mini-Mast control problem provided that modeling error does not dominate the problem formulation.

All the robust controllers synthesized and implemented on the Mini-Mast experiment use the four Bay 18 accelerometers for feedback. They all include $\mathcal{H}_{\infty}$ norm performance specifications on bays 10 and 18. In addition the reduced order five-mode model is used in the problem formulation, and the truncated structural modes of the full-order model are accounted for in the $\mu$-synthesis control designs via an additive uncertainty description. These results obtained with these designs are briefly discussed in the next section, and are treated in detail in [18].

\section{Robust CONTROLLER DESIGN AND IMPLEMENTATION}

In this section, we present experimental results of the vibration attenuation at bays 10 and 18 based on the design of a controller using only Bay 18 accelerometers as sensors. The control objective for the Mini-Mast structure is to attenuate structural vibration at bays 10 and 18 due to input disturbances at bay 9 . The torque wheel actuators used for control are also located at bay 18. A more detailed description of the control design can be found in [17] and [18].

A block diagram of the problem formulation is shown in Fig. 10. The control design model, Mini-Mast, consists of the first five modes of the Mini-Mast structure. There are two sources of uncertainty. Uncertainty in the torque wheel actuators models is represented as a constant, 2\% input multiplicative uncertainty to the actuator. This level was determined to be the most suitable input uncertainty description from a performance versus robustness study performed on the Mini-Mast facility. An additive uncertainty model is included to account for the high-frequency dynamics of the Mini-Mast structural model neglected in the design model and neglected actuator and sensor dynamics. The unmodeled dynamics correspond to the 23 modes between $92 \mathrm{rad} / \mathrm{s}$. to 461 $\mathrm{rad} / \mathrm{s}$. The additive uncertainty weight requires a prescribed level of roll off of the loop gain, which in effect requires the unmodeled high-frequency structural modes to be gain stablized by the controllers

$$
\mathrm{W} \_ \text {add }=\frac{19.5\left(s^{2}+25.46 s+324\right)\left(s^{2}+42.43 s+900\right)}{\left(s^{2}+1414 s+100000\right)\left(s^{2}+282.84 s+40000\right)}
$$



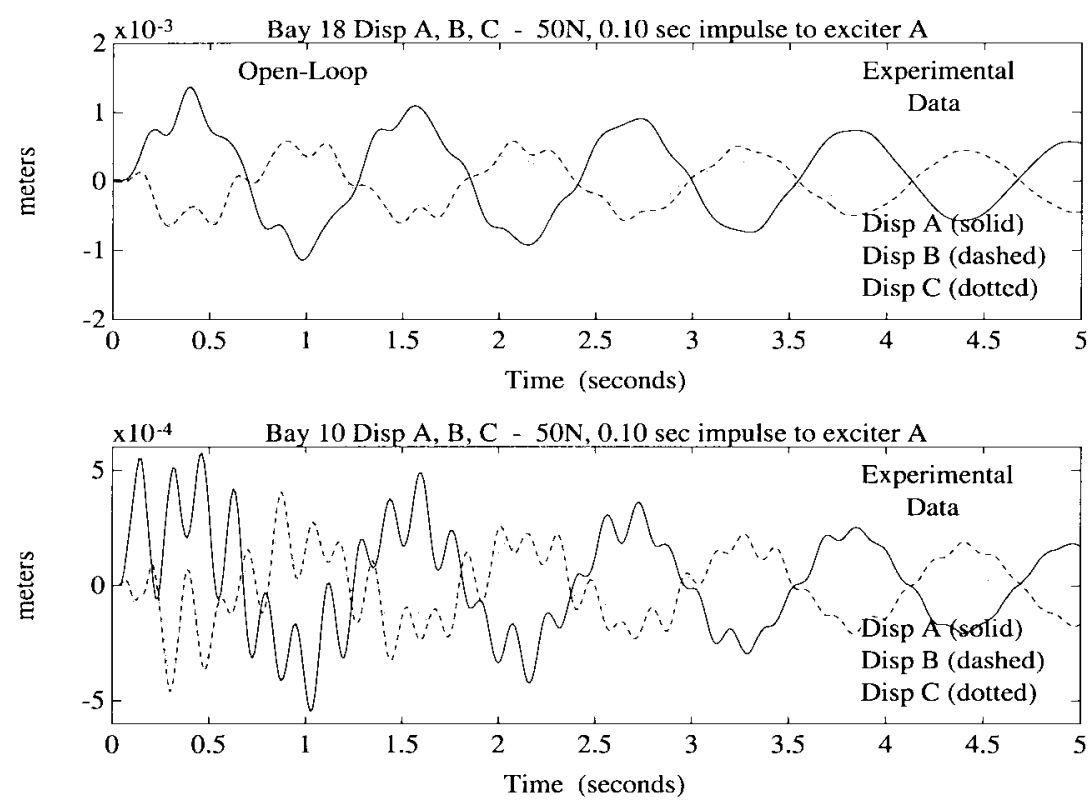

Fig. 11. Mini-Mast open-loop experimental displacement responses of bay 18 and 10 .
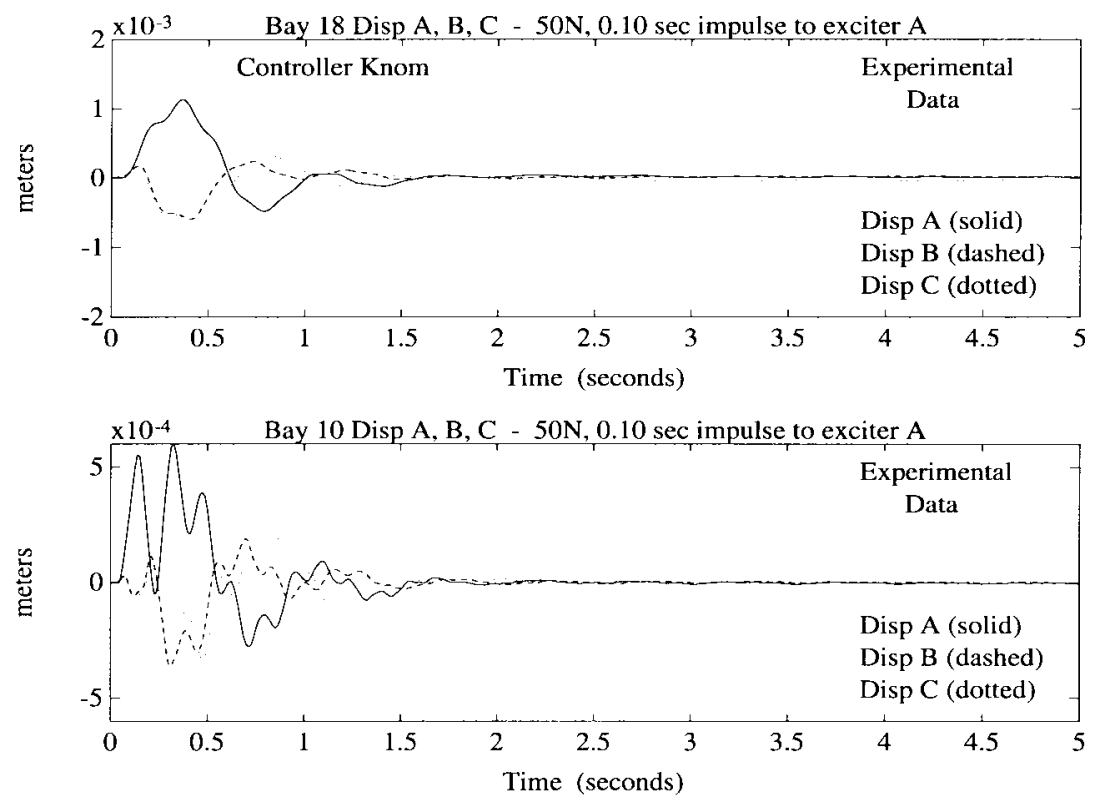

Fig. 12. Experimental displacement responses of bay 18 and 10 with Knom implemented.

The performance specification of attenuating the vibration of the first five modes is introduced into the problem formulation by weights on bay 10 and 18 displacements. The disturbance enters the structure via exciters at bay 9 . A frequency domain weight, Dist_wt equal to $31 /(s+31)$, is used to model the $10 \mathrm{~N}, 0.1$ second impulse disturbance to each exciter. Perf_disp weights on bay 10 and bay 18 displacements are taken to be 4000 and $20000(s+.5) /(s+50)$, respectively. The performance objective corresponds to reducing the peaks in the transfer functions between the exciter inputs and displacement outputs. The weighting on the actuator command, Act_mag, is one. This is similar to allowing the torque actuators to supply a maximum torque of $\pm 8 \mathrm{Nm}$ to attenuate the $10-\mathrm{N}$, 0.1-s impulse disturbance. A block diagram description of the control problem formulation is shown in Fig. 10.

A 49-state controller was designed using D-K iteration and reduced to 34 states using balanced realization techniques [19], [24]-[27]. This controller, Knom, was implemented in discrete-time at $80 \mathrm{~Hz}$ on the Mini-Mast experiment. The Mini-Mast structure was disturbed with a 50-N, 0.1-s impulse into exciter $\mathrm{A}$ and the goal is to attenuate the response of the structure at bays 10 and 18. Comparison of the openloop response of the structure, Fig. 11, and the closed-loop response, Fig. 12, indicate that the controller significantly attenuates vibration at bay 10 and 18 . This controller also commanded less than $\pm 80 \mathrm{~N} / \mathrm{m}$ of torque from the torque 

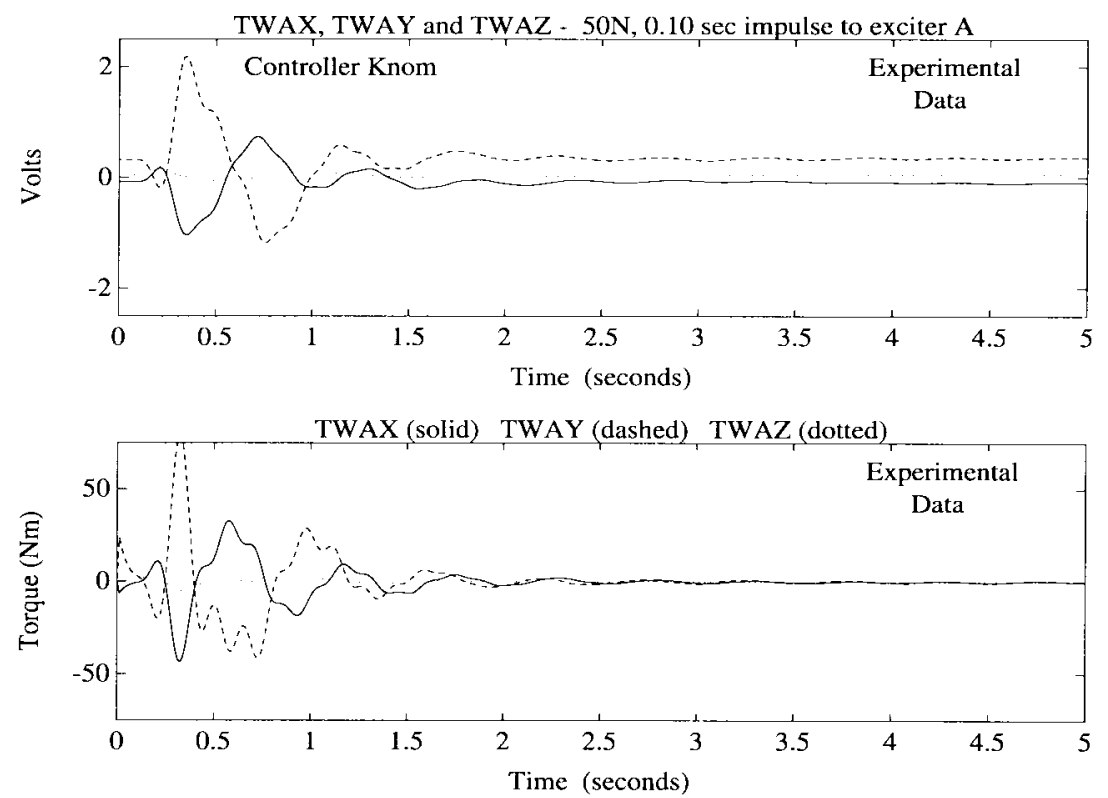

Fig. 13. Experimental torque wheel commands with Knom implemented.

wheel actuators during the experiment as seen in Fig. 13 [18]. Hence we have shown that the proposed method for sensor selection accurately predicted the ability of a controller to significantly attenuation the structural response using only bay 18 accelerometers as feedback sensors.

\section{SUMMARY}

$\mathcal{H}_{2}$ control design techniques were used to examine the effect of the type, placement, and number of feedback sensors on achievable closed-loop performance for the Mini-Mast structure. All the designs were studied theoretically, with the assumption of no modeling error in the problem formulation, although the results were validated using both full- and reduced order models. The $\mathcal{H}_{2}$ optimal designs presented in this paper were not intended to be implemented on the Mini-Mast facility, rather they were used to examine its fundamental properties and limitations.

The elegant separation structure of $\mathcal{H}_{2}$ optimal control was used to develop a closed-loop sensor selection methodology. The results indicated that for this problem one need only use bay 18 accelerometers for feedback control on the Mini-Mast structure, even with performance specifications on bays 10 and 18. The use of additional sensors cannot provide any significant performance improvement for this problem. The superiority of accelerometers over displacement sensors was also indicated, particularly in the noncolocated case. This is likely due to accelerometers observing the mid-frequency modes of the structure better than the displacement sensors.

The methodology proposed here for sensor selection is particularly suitable when the achievable robust performance level is close to the optimal nominal performance of the structure. In other words, these results are extendible to cases where the modeling error does not dominate the problem formulation. This criterion was found to hold true for the Mini-Mast structure, and a number of robust controllers were designed and implemented on the structure. All these controllers used only the four Bay 18 accelerometers for feedback, and yet were able to deliver high performance in practice on bays 10 and 18 .

\section{ACKNOWLEDGMENT}

The authors would like to thank Dr. K. Lim and Dr. P. Maghami for many useful discussions and K. Belvin, S. Tanner, J. Sulla, and R. Smith-Taylor for their assistance in carrying out the experiments.

\section{REFERENCES}

[1] W. Gawronski and K. B. Lim, "Balanced actuator and sensor placement for flexible structures," Int. J. Contr., vol. 65, no. 1, pp. 131-145, 1996.

[2] K. B. Lim, "Method for optimal actuator and sensor placement for large flexible structures," AIAA J. Guidance, Dynamics, Contr,, vol. 15, pp. 49-57, 1992.

[3] R. W. Longman and K. T. Alfriend, "Energy optimal degree of controllability and observability for regulator and maneuver problems," in Proc. 16th Annu. Conf. Inform. Sci. Syst., Princeton, N.J., Mar. 1982.

[4] Y. Kim and J. L. Junkins, "A measure of controllability for actuator placement," AIAA J. Guidance, Dynamics, Contr., vol. 14, no. 5, pp. 895-902, Sept.-Oct. 1991.

[5] K. B. Lim, "Disturbance rejection approach to actuator and sensor placement," AIAA J. Guidance, Dynamics, Contr., vol. 20, no. 1, pp. 203-204, 1997.

[6] W. H. Chen and J. H. Seinfeld, "Optimal location of process measurements," Int. J. Contr., vol. 21, no. 6, pp. 1003-1014, 1975.

[7] P. G. Maghami and S. M. Joshi, "Sensor-actuator placement for flexible structures," in Proc. 1990 Amer. Contr. Conf., San Diego, CA, May 23-25, 1990.

[8] _ _Sensor-actuator placement for flexible structures with actuator dynamics," in Proc. AIAA Guidance, Navigation, Contr. Conf., New Orleans, LA, 1991, pp. 46-54.

[9] R. E. Skelton and M. L. DeLorenzo, "Selection of noisy actuators and sensors for linear stochastic systems," J. Large-Scale Syst., Theory, Applicat., vol. 4, pp. 109-136, Apr. 1983.

[10] , "Space structure control design by variance assignment," AIAA J. Guidance, Dynamics, Contr., vol. 8, pp. 454-462, July-Aug. 1985.

[11] G. A. Norris and R. E. Skelton, "Selection of dynamic sensors and actuators in the control of linear systems," J. Dynamics Systems, Measurements, Contr., vol. 111, pp. 389-397, Sept. 1989. 
[12] R. E. Skelton and G. A. Norris, "Selection of noisy sensors and actuators in the presence of correlated noise," J. Contr. Theory Advanced Technol., vol. 2, pp. 102-111, 1989.

[13] J. N. Juang and G. Rodriguez, "Formulation and applications of large structure actuator and sensor placements," VPI/AIAA Symp. Dynamics Contr. Large Flexible Structures, 1979, pp. 247-262.

[14] E. G. Collins Jr., J. A. King, D. J. Philips, and D. C. Hyland, "High performance, accelerometer-based control of the Mini-Mast structure," AIAA J. Guidance, Contr., Dynamics, vol. 15, no. 4, pp. 885-892, 1992.

[15] S. E. Tanner, R. S. Pappa, J. L. Sulla, K. B. Elliot, R. Miserentino, J. P. Bailey, P. A. Cooper, B. L. Williams, and A. M. Bruner, Minimast CSI Testbed-User's Guide, NASA TM-102630, Mar. 1989.

[16] C. Hsieh, J. H. Kim, and R. E. Skelton, "Closed-loop lab tests of NASA's Mini-Mast," in Proc. Amer. Contr. Conf., May 1990, pp. 1435-1440.

[17] G. J. Balas and P. M. Young, "Control design for variations in structural natural frequencies," AIAA J. Guidance, Dynamics, Contr., vol. 18, no. 2, pp. 325-332, Mar.-Apr. 1995.

[18] G. J. Balas, P. Young, and J. C. Doyle, " $\mu$-based control design as applied to large space structures: Control design for the Minimast facility," NASA CSI/GI Final Rep., NAG-1-967, 1992.

[19] G. J. Balas, J. C. Doyle, K. Glover, A. K. Packard, and R. Smith, $\mu$ Analysis and Synthesis Toolbox: $\mu$-Tools and User's Guide, MUSYN Inc. and The Mathworks Inc., Dec. 1990.

[20] B. D. O. Anderson and J. B. Moore, Optimal Filtering. Englewood Cliffs, NJ: Prentice-Hall, 1989.

[21] K. Zhou, Robust and Optimal Control. Englewood Cliffs, NJ: PrenticeHall, 1996.

[22] J. C. Doyle, K. Glover, P. P. Khargonekar, and B. A. Francis, "Statespace solutions to standard $\mathrm{H}_{2}$ and $\mathrm{H}_{\infty}$ control problems," IEEE Trans. Automat. Contr., vol. 34, pp. 831-847, Aug. 1989.

[23] K. Glover and J. Doyle, "State-space formulae for all stabilizing controllers that satisfy an $H_{\infty}$ norm bound and relations to risk sensitivity," Syst. Contr. Lett., vol. 11, pp. 167-172, 1988.

[24] G. J. Balas and J. C. Doyle, "Control of lightly damped, flexible modes in the controller crossover region," AIAA J. Guidance, Dynamics, Contr., vol. 17, no. 2, pp. 370-377, Mar.-Apr. 1994.

[25] G. J. Balas and J. C. Doyle, "Robustness and performance tradeoffs in control design for flexible structures," IEEE Trans. Contr. Syst. Technol., vol. 2, pp. 352-361, Dec. 1994.

[26] G. J. Balas, P. Young, and J. C. Doyle, "The process of control design for the NASA Langley Minimast structure," in Amer. Contr. Conf., Boston, MA, 1991.

[27] A. Packard, J. C. Doyle, and G. J. Balas, "Linear, multivariable robust control with a $\mu$ perspective," ASME J. Dynamics, Measurements,
Contr., Special Edition on Control, vol. 115, no. 2b, pp. 426-438, June 1993.

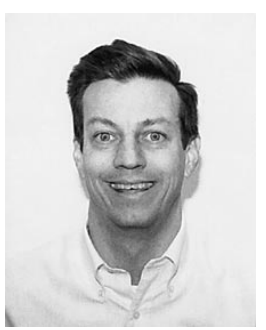

Gary J. Balas (S'89-M'89) received the B.S. and M.S. degrees in civil and electrical engineering from the University of California, Irvine, and the Ph.D. degree in aeronautics and astronautics from the California Institute of Technology, Pasadena, in 1990

Since 1990 he has been a faculty member in the Department of Aerospace Engineering and Mechanics at the University of Minnesota. From 1993 to 1995, he held a McKnight-Land Grant Professorship. Currently he is an Associate Professor and Codirector of the Control Science and Dynamical Systems Department at the University of Minnesota. He is a coorganizer and developer of the MUSYN Robust Control Short Course and the $\mu$-Analysis and Synthesis Toolbox used with MATLAB, and the President of MUSYN Inc. His research interests include control of flexible structures, aircraft, model validation, and industrial applications of robust control methods.

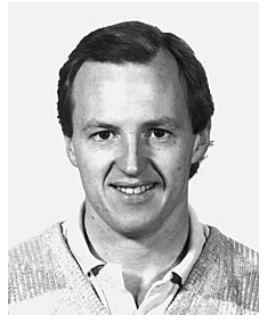

Peter Michael Young (S'90-M'92) was born in Wakefield, U.K., on September 21, 1962. He received the B.A. degree from Oxford University, Oxford, U.K, in 1985, the M.S. degree from the University of Florida, Gainesfille, in 1988, and the Ph.D. degree from California Institute of Technology, Pasadena, in 1993.

From 1985 to 1986 he was an Executive Engineer at British Telecom Research Laboratories, and from 1993 to 1995 he was a Postdoctoral Associate at Massachusetts Institute of Technology. Currently he is an Assistant Professor at Colorado State University, Ft. Collins. His research interests include the the development of advanced analysis and design techniques for large-scale uncertain MIMO systems, subject to both multiple uncertain parameters, and multiple dynamic uncertainties. He is also interested in the development of synthesis tools for robust controller design, and a number of specific application areas, which include structural vibration suppression, control of HVAC systems, power systems, hard disk drives, and haptic interfaces. 